\title{
The measurement of reactive oxygen species in human neat semen and in suspended spermatozoa: a comparison
}

\author{
Helena Fingerova, Ivana Oborna*, Jiri Novotny, Magda Svobodova, \\ Jana Brezinova and Lenka Radova
}

Address: Department of Obstetrics and Gynecology, University Hospital Olomouc, IP Pavlova 6, 77520 Olomouc, Czech Republic

Email: Helena Fingerova - hefi@centrum.cz; Ivana Oborna* - obornai@seznam.cz; Jiri Novotny - novotnj1@tunw.upol.cz; Magda Svobodova - svobodovam@fnol.cz; Jana Brezinova - janabrez@seznam.cz; Lenka Radova - lenka_radova@email.cz

* Corresponding author

Published: 27 October 2009

Reproductive Biology and Endocrinology 2009, 7:118 doi:10.1186/1477-7827-7-118

This article is available from: http://www.rbej.com/content/7/1/1 18

(c) 2009 Fingerova et al; licensee BioMed Central Ltd.

This is an Open Access article distributed under the terms of the Creative Commons Attribution License (http://creativecommons.org/licenses/by/2.0), which permits unrestricted use, distribution, and reproduction in any medium, provided the original work is properly cited.
Received: 7 August 2009

Accepted: 27 October 2009

\begin{abstract}
Background: It is generally accepted that oxidative stress is an important factor in male infertility because it may impair the physiological function of spermatozoa at the molecular level. Nevertheless, although several approaches have been reported, the imbalance between production of reactive oxygen species (ROS) and activity of the antioxidant defense system in semen is difficult to investigate and remains poorly understood.
\end{abstract}

Methods: This study compares measurement of ROS production in neat semen and in washed spermatozoa obtained from the same ejaculate, and suspended in phosphate buffered saline using exactly the same luminol-mediated chemiluminescence method. Ninety one samples were obtained from males of infertile couples and 34 from volunteers with proven fertility.

Results: As expected, ROS levels were markedly lower in neat semen than in washed spermatozoa suspensions where seminal plasma with its potent antioxidant capacity was removed. In the cases of both neat semen and washed spermatozoa, ROS production was lowest in samples from normozoospermic males and highest in samples containing more than half million peroxidasepositive leukocytes per milliliter. For all samples, there was a significant positive correlation between ROS production by neat semen and that by washed spermatozoa suspension.

Conclusion: Measurement of ROS production in neat semen better reflects actual oxidative status because it detects only the overproduction of ROS which are not effectively scavenged by antioxidant capacity of seminal fluid. The results of our study show a good commutability of both measurements for identification of semen samples with high ROS production. The measurement in neat semen is even less time consuming and therefore easier to implement into laboratory routine.

\section{Background}

Reactive oxygen and reactive nitrogen species (ROS and RNS), a group of highly reactive oxidants, most of which contain unpaired electrons, are usually known as ROS or free radicals. At physiological levels they have important roles in metabolism in all aerobic organisms. However, excessive ROS production which can not be effectively controlled by antioxidants leads to oxidative stress (OS) 
which has been linked to many pathological processes, including male infertility [1-3]. In the genital tract, low levels of ROS are necessary for normal function of human spermatozoa [4], including their capacitation, acrosome reaction and sperm-oocyte fusion. On the other hand, excessive OS may cause lipoperoxidation of sperm membranes resulting in DNA damage and sperm apoptosis. Standard semen analysis is often inadequate to explain conception failure as the routine microscopic evaluation can not reveal subtle disorders at the molecular level which may be caused by OS [5]. This situation often leads to diagnosis of idiopathic infertility. Moreover, there is some degree of overlap in sperm parameters between fertile and infertile males [6].

Although the importance of seminal OS assessment has already been advocated for semen analysis by WHO Laboratory Manual 1999 [7], only a few centers worldwide have so far developed methods for indirect measurement of ROS production in semen. ROS production in human spermatozoa was first measured in washed spermatozoa suspended in phosphate buffered saline (PBS) to the concentration of 20 millions per milliliter. The chemiluminescent signal induced by the addition of luminol was measured integrally and expressed in cpm $[2,8,9]$. We implemented this method in the University Hospital in Olomouc in 2002, to our knowledge for the first time in the Czech Republic. The weakness of this approach is that seminal plasma with its powerful total antioxidant capacity (TAC) is removed prior to measurement. Later, as a better predictor of oxidative sperm damage, the ROS/TAC score was introduced [10]. Unfortunately, both ROS and TAC measurements are subject to various methodological problems. ROS measurement by luminol-induced chemiluminescence in neat semen was first reported by Allamaneni et al. [11]. Since the beginning of 2007 we have been measuring ROS simultaneously in neat ejaculate and in spermatozoa suspension in PBS, i.e. washed semen. The aim of our study was to compare their clinical usefulness in the evaluation of the male factor infertility and to evaluate the commutability between the ROS measurement in washed and neat semen.

\section{Methods \\ Study group}

Within the period of two years, 91 semen samples were obtained from 85 males from couples asking for enrollment into infertility treatment. The only inclusion criterion was a sperm concentration over $20 \times 10^{6} / \mathrm{ml}$ to ensure a sufficient precision of ROS measurement. Another 34 semen samples from 22 volunteers with proven fertility served as a control group. The time span between the repeated semen samples in 18 subjects was at least 6 months and in this sense was considered as an independent variable. The average age of all subjects was $33 \pm 5$ years and there was no significant difference in age between the evaluated groups. The study was approved by the Ethical Board of the Palacky University Medical Faculty in Olomouc and all subjects signed written consent.

\section{Study design}

Ejaculates were obtained by masturbation following three to four days of sexual abstinence. After liquefaction $\left(37^{\circ} \mathrm{C}, 30 \mathrm{~min}\right)$, standard semen analysis was performed according to the WHO Manual [7]. One aliquot of $0.5 \mathrm{ml}$ of liquefied neat semen was used for immediate ROS measurement and another $1 \mathrm{ml}$ aliquot of semen was taken for preparation of spermatozoa suspension in PBS and following ROS measurement by the same luminolinduced chemiluminescence method [12].

\section{ROS detection by chemiluminescent assay in sperm suspension in PBS}

The method was described in detail previously [12]. Briefly, the liquefied semen was centrifuged at $300 \mathrm{~g}$ for 7 min, seminal plasma was removed and the pellet of cells was washed in PBS (isotonic solution, $\mathrm{pH}=7.4$ ) and spun again and decanted. Washed cells were suspended in PBS to adjust sperm concentration to $1.25 \times 10^{6} / \mathrm{ml}$. ROS production was measured after addition of $10 \mu \mathrm{l}$ of $5 \mathrm{mM}$ freshly prepared solution of luminol (5-amino-2,3-dihydro-1,4-phthalazinedione, Sigma Chemical Co., St. Louis, MO, USA) in dimethylsulphoxide (DMSO, Sigma Chemical Co.) to $400 \mu \mathrm{l}$ of spermatozoa suspension. A tube containing $400 \mu \mathrm{l}$ of PBS and $10 \mu \mathrm{l}$ of luminol solution served as a blank. Chemiluminescence was measured integrally for 15 minutes using the Digene DCR-1 single detector luminometer (Digene Diagnostics, Inc., Gaithersburg, MD, USA). Results were expressed in relative light units (RLU) per minute and per $20 \times 10^{6}$ spermatozoa.

\section{ROS detection by chemiluminescent assay in neat semen}

The ROS production in $400 \mu$ l of liquefied neat semen was measured after addition of $10 \mu \mathrm{l}$ of $5 \mathrm{mM}$ solution of luminol in DMSO. A tube containing $10 \mu \mathrm{l}$ of $5 \mathrm{mM}$ luminol solution in DMSO was used as a blank. Chemiluminescence was measured for $15 \mathrm{~min}$ as described above. The RLU/min were then recalculated according to the original spermatozoa concentration in semen sample and expressed as RLU/min per $20 \times 10^{6}$ spermatozoa.

\section{Statistical analysis}

Statistical calculations were performed using Statistica 8 (StatSoft CR) [13]. Mann-Whitney test was used to compare data between groups. Spearman correlation coefficient was used to evaluate interdependence between the ROS production found in neat semen and that found in sperm suspension. Logarithmic transformation was used 
for this purpose due to a very large range of inter-individual values. Statistical significance was set at $\mathrm{p}<0.05$ for all tests.

\section{Results \\ Semen analysis}

Semen samples were classified according to the WHO Manual [7] as normozoospermic when sperm concentration was $\geq 20 \times 10^{6} / \mathrm{ml}$, motility $\geq 50 \%$, morphology $\geq$

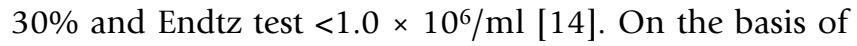
microscopic evaluation, 39 semen samples from males of infertile couples were normozoospermic (NS-males) and 52 semen samples (SA-males) revealed various semen abnormalities which included 14 asthenozoo-, 29 astenoteratozoo-, 4 teratozoo- and 5 samples with leukocyte count over $0.5 \times 10^{6} / \mathrm{ml}$ including three leukocytospermias. Semen samples $(n=34)$ of fertile volunteers (FVmales) served as a control. With regard to our previous finding [15] that a concentration of peroxidase positive leukocytes in Endtz test higher than $0.5 \times 10^{6} / \mathrm{ml}$ may significantly contribute to ROS production in semen, we have further subdivided the SA group into Endtz test negative samples (SA EN subgroup, $\mathrm{n}=27$ ), samples with leukocyte concentrations below $0.5 \times 10^{6} / \mathrm{ml}$ (SA EL subgroup, $\mathrm{n}=20$ ) and over $0.5 \times 10^{6} / \mathrm{ml}$ (SA EH subgroup, $n=5$ ). The data on age and semen parameters of all subjects enrolled in the study are shown in Table 1.

\section{ROS detection in neat semen}

As seen in Table 2, the lowest ROS production in neat semen was found in the fertile volunteers (FV), as well as in normozoospermic males from infertile couples (NS) and in leukocyte-free samples from males with semen abnormalities (SA EN). Significantly higher ROS production compared to fertile volunteers was found in SA EL samples $(\mathrm{p}<0.005)$ and the highest ROS production was found in SA EH samples $\left(\mathrm{p}<10^{5}\right)$.

\section{ROS detection in sperm suspension in PBS}

ROS production in sperm suspension was generally higher than that in neat semen (up to $1 \times 10^{7} \mathrm{RLU} / \mathrm{min}$ per $20 \times 10^{6}$ spermatozoa). There was no difference in ROS production between FV, NS, SA EN and SA EL males.
A significant difference $\left(\mathrm{p}<10^{-5}\right)$ was found only in the SA EH group.

There was a significant positive correlation $(\mathrm{r}=0.476, \mathrm{p}<$ $1 \times 10^{-6}$ ) between the ROS levels in neat semen and those in sperm suspension (Fig 1). Most of the FV and NS samples were closer to the regression line and prevalently below it, while the SA samples were much more widely scattered along the regression line in both directions. On the contrary, the SA EH samples were all above the regression line.

\section{Discussion}

Since the appearance of the first reports in the early nineteen nineties $[1,2,16,17]$, the role of OS in the pathophysiology of male infertility has been gradually accepted. The importance of seminal ROS production has been already stressed in the WHO Manual (1999) and several methods of ROS detection in semen have been reported $[8,9,12,18]$. Nevertheless, reliable and reproducible methods of ROS measurement in semen for routine clinical use are still missing. Such method(s) would be a useful tool in the diagnosis of male infertility and in the selection of patients who would benefit from antioxidant treatment [3,18-22].

Measurement of the level of OS in ejaculate arising from imbalance between ROS production and the capacity of the complex antioxidant defense system is extremely difficult. The direct measurement of free radicals is practically impossible. The indirect methods have either used lucigenin or luminol mediated chemiluminescence in spermatozoa suspension [1], or they have to rely on the detection of some stable oxidized end-products, the so called biomarkers of OS, mostly in body fluids [23]. Luminol mediated luminescence is preferred as it can detect the sum of several important intra- and extra-cellular ROS including hydrogen peroxide, superoxide and hydroxyl radicals $[2,9,16]$.

Unfortunately, there are some limitations to ROS measurement in spermatozoa suspension in PBS. The absolute values are expressed either in count per minute (cpm) or

Table I: Semen parameters of all subjects

\begin{tabular}{lccc}
\hline Number of samples & $\mathbf{F V}$ & $\mathbf{N S}$ & $\mathbf{S A}$ \\
$\mathbf{n = 3 4}$ & $\mathbf{n = 5 2}$ & $3.3 \pm 1.3$ \\
\hline Semen volume $(\mathrm{ml})$ & $2.8 \pm 1.3$ & $3.2 \pm 1.4$ & $195 \pm 99$ \\
Total sperm count $\left(\times 10^{6}\right)$ & $169 \pm 104$ & $65.9 \pm 31.1$ & $50.9 \pm 25.0$ \\
Sperm concentration $(\times 106 / \mathrm{ml})$ & $64.5 \pm 29.7$ & $53.8 \pm 7.7$ & $37.3 \pm 8.8$ \\
Motility $(\%)$ & $44.2 \pm 10.2$ & $38.1 \pm 5.8$ & $25.1 \pm 8.2$ \\
Normal morphology $(\%)$ & $31.5 \pm 8.6$ & &
\end{tabular}

FV - fertile volunteers, NS - normozoospermic males, SA - males with semen abnormalities.

Values are expressed as mean \pm SD 
Table 2: Differences of ROS production in neat semen and in spermatozoa suspension between fertile volunteers and subgroups of males from infertile couples

\begin{tabular}{|c|c|c|c|c|c|}
\hline groups & $\mathbf{n}$ & ROS $\left(R L U \times 10^{3}\right)$ in neat semen & ap & ROS $\left(R L U \times 10^{4}\right)$ in spermatozoa suspension & ${ }^{b} \mathbf{p}$ \\
\hline $\mathrm{FV}$ & 34 & $0.26(0.12 ; 0.55)$ & & $3.5(1.3 ; 13.0)$ & \\
\hline NS & 39 & $0.31(0.12 ; 1.12)$ & $<0.52$ & $2.4(1.0 ; 20.2)$ & $<0.91$ \\
\hline SA & 52 & $1.1(0.19 ; 4.1)$ & $<0.005$ & $9.3(1.4 ; 118.3)$ & $<0.055$ \\
\hline SA subgroups of SA EN & 27 & $0.24(0.15 ; 1.30)$ & $<0.27$ & $3.9(0.9 ; 28.2)$ & $<0.1$ \\
\hline SA EL & 20 & $1.14(0.36 ; 4.2)$ & $<0.005$ & II.0 (2.0; 107.3) & $<0.76$ \\
\hline SA EH & 5 & $32.3(7.40 ; 55.8)$ & $<10^{-5}$ & $385.0(170.0 ; 625.0)$ & $<10^{-5}$ \\
\hline
\end{tabular}

Values are presented as median ( $25^{\text {th }} ; 7^{\text {th }}$ percentile). The ${ }^{\mathrm{a}} \mathrm{p}$ and ${ }^{\mathrm{b}} \mathrm{P}$ values designate the differences in ROS production in subgroups of males from infertile couples versus FV in neat semen and in spermatozoa in PBS suspension, respectively. A value of $p<0.05$ was considered significant by Mann-Whitney $U$ test.

FV - fertile volunteers, NS - normozoospermic males, SA - males with semen abnormalities,

SA EN - subgroup of SA with negative Endtz test, SA EL - subgroup of SA with Endtz test $<0.5 \times 106 / \mathrm{ml}$, SA EH - subgroup of SA with Endtz test $>0.5 \times 10^{6} / \mathrm{ml}$

RLU per minute, and moreover, may differ with respect to the sensitivity and type of luminometer used. ROS production by spermatozoa suspended in PBS declines with time, therefore the measurement should be performed within one hour of obtaining semen sample [9]. There is also a possibility of an artificial increase in ROS production caused by repeated centrifugation during the preparation of the spermatozoa suspension [14]. All this makes it difficult to compare results reported from different settings. Another, even more important drawback of the measurement of ROS in washed spermatozoa, is the fact that they are deprived of their natural antioxidant environment, seminal plasma. The ROS/TAC score [10], thought to assess only the excess of ROS not scavenged by seminal plasma antioxidants, introduces another variable which may also be prone to analytical error. According to our experience [24], the TAC of seminal plasma measured by the TAS Randox ${ }^{\circledast}$ method varied to a much smaller extent in contrast to ROS production because it measured only non-enzymatic antioxidants.

The determination of ROS production in neat semen is a better solution. It avoids centrifugation and washing procedures and shortens the time lag between semen collection and ROS measurement. Moreover, it can be expected to measure only the excess ROS which are not scavenged by seminal plasma antioxidants and thus directly identify samples with OS. In our setting, the ROS levels in neat semen were lowest in samples from fertile volunteers, normozoospermic men and leukocyte-free samples from men with semen abnormalities. ROS levels were significantly higher in samples with peroxidase-positive leukocyte concentrations $<0.5 \times 10^{6} / \mathrm{ml}$ and the highest in samples with leukocyte concentrations $>0.5 \times 10^{6} / \mathrm{ml}$. The corresponding results measured in spermatozoa suspension in PBS were markedly higher, in some cases up to two orders of magnitude. A significantly higher ROS production was detected only in samples from SA males with leukocyte concentrations $>0.5 \times 10^{6} / \mathrm{ml}$.
Our findings of a reasonable commutability of measuring ROS in washed and neat semen well agree with the first reported comparison of luminol-mediated chemiluminescent measurement of ROS production in neat semen versus spermatozoa suspensions in PBS [11]. Allamameni et al. evaluated semen from 34 semen donors and 44 patients with abnormal semen parameters. The ROS production in neat semen of donors was about five times lower than the respective ROS levels in spermatozoa suspension. The levels measured in neat semen of patients with abnormal semen were significantly higher than in healthy donors. A later study of Athayde et al. included semen samples from 114 fertile Brazilian men seeking voluntary sterilization by vasectomy and 47 subfertile males [25]. In samples without leukocytes, the threshold for normality of ROS in neat semen samples was set at $0.55 \times 10^{4} \mathrm{cpm}$ per $20 \times 10^{6}$ spermatozoa; almost 20 times lower than that in washed spermatozoa in PBS. A recent study by this group [26] reported the median and interquartile range of seminal ROS levels in neat semen samples of 78 vasectomy candidates younger than 40 years as $0.29(0.18,0.58) \times 10^{4} \mathrm{cpm}$ per $20 \times 10^{6}$ spermatozoa, which agrees well with our findings in Czech fertile volunteers, that is $0.26(0.12,0.55) \times 10^{3} \mathrm{RLU} / \mathrm{min}$ per $20 \times 10^{6}$ spermatozoa, even though they were obtained on a different population and using a different luminometer.

This study, to our knowledge the first one in the Czech Republic, has proved that a significant positive correlation exists between ROS levels in neat semen and that in spermatozoa suspension in PBS, in which the antioxidant capacity of seminal plasma is absent. This suggests that the individual total antioxidant capacity of seminal plasma may vary to a lesser extent than the ROS produced by spermatozoa and/or activated leukocytes.

\section{Conclusion}

We conclude that ROS measurement by luminol-mediated chemiluminescence in neat semen provides a better 


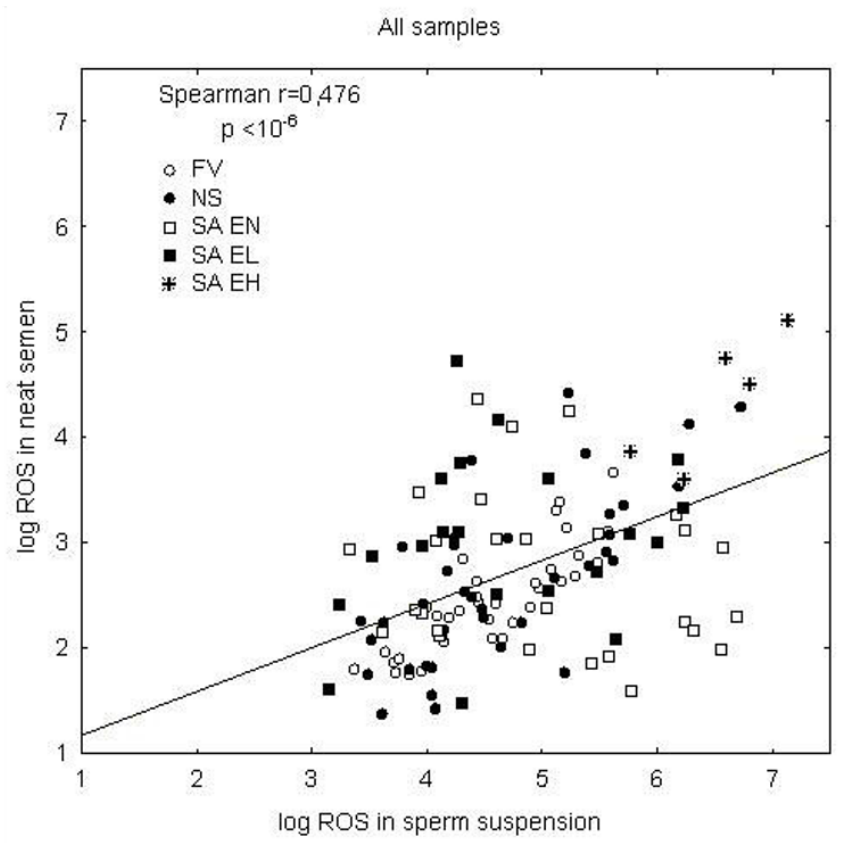

Figure I

Correlation between $\log _{10}$ ROS in neat semen and $\log _{10}$ ROS in sperm suspension in PBS in all samples. Individual groups are differentiated by symbols.

assessment of the actual level of OS than the measurement in washed spermatozoa. The ROS determination in neat semen is rapid, simple and can be used also for ROS measurement in severe oligozoospermia [27]. Though the role of OS as a cause of male infertility has been generally accepted, ROS measurement has not yet been routinely used in clinical infertility treatment. There is still a lack of a single standardized measure of OS [3]. Laboratories which want to introduce OS evaluation by this chemiluminescence method will have to establish and validate their own reference ranges. Further research is also needed to elucidate if and how ROS production in neat semen correlates with other surrogate OS markers $[3,23]$.

\section{Competing interests}

The authors declare that they have no competing interests.

\section{Authors' contributions}

HF wrote the paper, IO designed the study, recruited study subjects and controls, provided clinical information and gave final approval, JN perfomed chemiluminesce measurements, JB and MS performed semen analysis, samples preparation and data collection, LR performed statistical analysis. All authors read and approved the final manuscript.

\section{Acknowledgements}

This study was supported by the Internal Grant Agency of the Czech Ministry of Health IGA MZd AI 862I-5

\section{References}

I. Aitken RJ, Clarkson JS, Fishel S: Generation of reactive oxygen species, lipid peroxidation, and human sperm function. Biol Reprod 1989, 41: I83-197

2. Sharma RK, Agarwal A: Role of reactive oxygen species in male infertility. Urology 1996, 48:835-850.

3. Tremellen $\mathrm{K}$ : Oxidative stress and male infertility - a clinical perspective. Hum Reprod Update 2008, 3:243-258.

4. de Lamirande E, Jiang H, Zini A, Kodama H, Gagnon C: Reactive oxygen species and sperm physiology. Rev Reprod 1997, 2:48-54.

5. Wathes DC, Abayasekara DRE, Aitken J: Polyunsaturated fatty acids in male and female reproduction. Biol Reprod 2007, 77: $190-201$.

6. Aziz N, Agarwal A: Evaluation of sperm damage: beyond the World Health Organization criteria. Fertil Steril 2008, 90:484-485

7. WHO laboratory manual for the examination of human semen and sperm-cervical mucus interaction. 4th edition. Cambridge University Press; 1999.

8. Ochsendorf FR, Thiele J, Fuchs J, Schuttau H, Freisleben HJ, Buslau M, Milbradt R: Chemiluminescence in semen of infertile men. Andrologia 1994, 26:289-293.

9. Kobayashi H, Gil-Guzman E, Mahran AM, Sharma RK, Nelson DR, Thomas AJ Jr, Agarwal A: Quality control of reactive oxygen species measurement by luminol-dependent chemiluminescence assay. J Androl 200I, 22:568-574.

10. Sharma RK, Pasqualotto FF, Nelson DR, Thomas AJ Jr, Agarwal A: The reactive oxygen species - total antioxidant capacity score is a new measure of oxidative stress to predict male infertility. Hum Reprod 1999, I 4:2801-2807.

II. Allamaneni SS, Agarwal A, Nallella KP, Sharma RK, Thomas AJ Jr, Sikka SC: Characterization of oxidative stress status by evaluation of reactive oxygen species levels in whole semen and isolated spermatozoa. Fertil Steril 2005, 83:800-803.

12. Novotny J, Oborna I, Brezinova J, Svobodova M, Hrbac J, Fingerova H: The occurrence of reactive oxygen species in semen of males from infertile couples. Biomed Pap 2003, I47:173-176.

13. StatSoft CR [http://www.statsoft.cz]

14. Shekarriz M, DeWire DM, Thomas AJ Jr, Agarwal A: A method of human semen centrifugation to minimize the iatrogenic sperm injuries caused by reactive oxygen species. Eur Urol 1995, 28:3|-35.

15. Oborna I, Fingerova H, Novotny J, Brezinova J, Svobodova M, Aziz N: Reactive oxygen species in human semen in relation to leukocyte contamination. Biomed Pap 2009, I 53:53-58.

16. Aitken RJ, Buckingham DW, West KM: Reactive oxygen species and human spermatozoa: analysis of the cellular mechanisms involved in luminol- and lucigenin-dependent chemiluminescence. J Cell Physiol 1992, I 5 I:466-477.

17. Griveau JF, Le Lannou D: Reactive oxygen species and human spermatozoa, physiology and pathology. Int J Androl 1997, 20:61-69.

18. Agarwal A, Sharma RK, Nallella KP, Thomas JA Jr, Alvarez JG, Sikka SC: Reactive oxygen species as an independent marker of male factor infertility. Fertil Steril 2006, 86:878-885.

19. Deepinder F, Cocuzza M, Agarwal A: Should seminal oxidative stress measurement be offered routinely to men presenting for infertility evaluation? Endocr Prac 2008, I 4:484-49।.

20. Agarwal A, Nallella KP, Allamaneni SS, Said TM: Role of antioxidants in treatment of male infertility: an overview of the literature. Reprod Biomed Online 2004, 8:616-627.

21. Dokmeci D: Oxidative stress, male infertility and the role of carnitines. Folia Med (Plovdiv) 2005, 47:26-30.

22. Goyal A, Chopra M, Lwaleed BA, Birch B, Cooper AJ: The effects of dietary lycopene supplementation on human seminal plasma. BJU Int 2007, 99:|456-1460.

23. Tsukahara $\mathrm{H}$ : Biomarkers for oxidative stress. Clinical application in pediatric medicine. Curr Med Chem 2007, I 4: I-I3.

24. Fingerova H, Novotny J, Barborik J, Brezinova J, Svobodova M, Krskova M, Oborna I: Antioxidant capacity of seminal plasma measured by TAS Randox ${ }^{\circledR}$. Biomed Pap 2007, I 5 I:37-40.

25. Athayde KS, Cocuzza M, Agarwal A, Krajcir N, Lucon AM, Srougi M, Hallak J: Development of normal reference values for seminal Reactive Oxygen Species and their correlation with leuko- 
cytes and semen parameters in a fertile population. J Androl 2007, 28:613-620.

26. Cocuzza M, Athayde KS, Agarwal A, Sharma RK, Pagani R, Lucon AM, Srougi M, Hallak J: Age-related increase of reactive oxygen species in neat semen in healthy fertile men. Urology 2008, 7I:49I-494.

27. Desai N, Sharma RK, Makker K, Sabanegh E, Agarwal A: Physiologic and pathologic levels of reactive oxygen species in neat semen of infertile men. Fertil Steril 2008.

Publish with Bio Med Central and every scientist can read your work free of charge

"BioMed Central will be the most significant development for disseminating the results of biomedical research in our lifetime. " Sir Paul Nurse, Cancer Research UK

Your research papers will be:

- available free of charge to the entire biomedical community

- peer reviewed and published immediately upon acceptance

- cited in PubMed and archived on PubMed Central

- yours - you keep the copyright

Submit your manuscript here:

http://www.biomedcentral.com/info/publishing_adv.asp 